\title{
Seeing the Phantom: A Functional Magnetic Resonance Imaging Study of a Supernumerary Phantom Limb
}

\author{
Asaid Khateb, PhD, ${ }^{1-3}$ Stéphane R. Simon, PhD, ${ }^{4}$ Sebastian Dieguez, MSc, ${ }^{5}$ François Lazeyras, PhD, ${ }^{4}$ \\ Isabelle Momjian-Mayor, MD, ${ }^{2}$ Olaf Blanke, Pr MD, ${ }^{2,5}$ Theodor Landis, $\operatorname{Pr~MD,~}{ }^{2,3}$ Alan J. Pegna, PhD, ${ }^{1-3}$ and \\ Jean-Marie Annoni, Pr MD $\mathrm{MD}^{2,3}$
}

Objective: Supernumerary phantom limb (SPL) is a rare neurological manifestation where patients with a severe stroke-induced sensorimotor deficit experience the illusory presence of an extra limb that duplicates a real one. The illusion is most often experienced as a somesthetic phantom, but rarer SPLs may be intentionally triggered or seen. Here, we report the case of a left visual, tactile, and intentional SPL caused by right subcortical damage in a nondeluded woman.

Methods: Using functional magnetic resonance imaging, we investigated the multimodal nature of this phantom, which the patient claimed to be able see, use, and move intentionally. The patient participated in a series of sensorimotor and motor imagery tasks involving the right, the left plegic, and the SPL's hand.

Results: Right premotor and motor regions were engaged when she imagined that she was scratching her left cheek with her left plegic hand, whereas when she performed the same task with the SPL, additional left middle occipital areas were recruited. Moreover, comparison of responses induced by left cheek (subjectively feasible) versus right cheek scratching (reportedly unfeasible movement) with the SPL demonstrated significant activation in right somesthetic areas.

Interpretation: These findings demonstrate that intentional movements of a seen and felt SPL activate premotor and motor areas together with visual and sensory cortex, confirming its multimodal dimension and the reliability of the patient's verbal reports. This observation, interpreted for cortical deafferentation/disconnection caused by subcortical brain damage, constitutes a new but theoretically predictable entity among disorders of bodily awareness.

Ann Neurol 2009;65:698-705

Phantom limbs are usually described after amputation of extremities and can be defined as "the persistent experience of the postural and motor aspects of a limb after its physical loss." 1 In hemiplegic patients after stroke, a similar phenomenon can occasionally occur, consisting in the experience of an additional limb. ${ }^{2-5}$ Much less frequent than amputation phantoms, this phenomenon, generally called supernumerary phantom limb (SPL), refers to "the awareness of having an extra limb in addition to the regular set of two arms and two legs." Compared with postamputation phantoms, which are overwhelmingly criticized by the patient as an illusory feeling, SPLs are sometimes part of delusional syndromes involving reduplications of body parts or "pseudopolymyelia." On other instances, the phenomenon can be more selective and the patient may retain a critical attitude toward his experience of an "extra limb." These generally involve focal subcortical lesions, and must be differentiated from patients affected by multiple delusional reduplications and suffering from larger cortical predominantly right lesion. ${ }^{7}$ Phenomenologically, the literature suggests a large spectrum of features. In most cases, the SPL has only somesthetic characteristics and cannot be seen or purposefully moved. ${ }^{8}$ SPLs with motor components mostly involve anarchic, uncontrollable movements, very restricted ones, ${ }^{10}$ or mirror automatically the healthy limb's movements. ${ }^{11,12}$ To our knowledge, only one case with a highly controllable and purposeful SPL has been reported and described as an "intentional motor phantom limb." 13 There are scattered reports of involvement of other modalities concomitantly to a
From the ${ }^{1}$ Laboratory of Experimental Neuropsychology and ${ }^{2}$ Department of Neurology, Geneva University Hospitals and Faculty of Medicine; ${ }^{3}$ Faculty of Medicine, Geneva Neuroscience Centre; ${ }^{4}$ Center for Biomedical Imaging and Department of Radiology, Geneva University Hospitals and Faculty of Medicine, Geneva; and ${ }^{5}$ Laboratory of Cognitive Neuroscience, Brain Mind Institute, Ecole Polytechnique Fédérale de Lausanne, Lausanne, Switzerland.

Address correspondence to Dr Khateb, Laboratory of Experimental Neuropsychology, Geneva University Hospitals, 24, rue Micheli-duCrest, 1211 Geneva 14, Switzerland. E-mail: asaid.khateb@hcuge.ch
Potential conflict of interest: Nothing to report.

Additional Supporting Information may be found in the online version of this article.

Received Sep 2, 2008, and in revised form Dec 19. Accepted for publication Jan 16, 2009.

Published in Wiley InterScience (www.interscience.wiley.com). DOI: 10.1002/ana.21647 
somesthetic SPL in the literature. A few patients reported a visual component to their SPL, saying they could see it. ${ }^{2,4,14-19}$ Still other cases were described with tactile components, with patients reporting that they can actually feel objects or body parts with their phantom. ${ }^{12,14,20-23}$ These intentional and visual aspects, although rare and poorly detailed, indicate that multimodal mechanisms might be involved in SPLs.

Given the abundance of components that might constitute the phenomenology of SPLs, different neural mechanisms have been proposed. Classic principles emphasize a dissociation between the previously established sensorimotor representations and the lesioninduced change in communication between the brain and the paralyzed limb (see Brugger's article ${ }^{5}$ ). A deficit in the representation of space, ${ }^{6}$ disordered realitytesting abilities, ${ }^{2,24}$ or failed updating of motor commands ${ }^{25}$ have also been proposed for SPLs.

Of special interest for the comprehension of the brain mechanisms of SPL are case studies using functional magnetic resonance imaging (fMRI). A recent study assessing the neural correlates of intentional movements of an SPL in a patient with subcortical stroke suggested an abnormal reorganization within the motor system. This hypothesis was based on the observation that SPL's movements were associated with increased fMRI responses in motor areas and in the bilateral basal ganglia-thalamus. ${ }^{13}$ The authors propose that cortical pathways during such movements "may reflect an abnormal closed-loop functioning of the thalamocortical system" caused by the interruption of thalamic sensory afferences. ${ }^{13}$ In another patient experiencing an SPL after a frontomesial stroke, the motor system was implicated in the illusion but at a more integrative level. ${ }^{26}$ Functional activations were reported in the right internal supplementary motor area during the presence of the SPL. The authors thus proposed that an uncontrolled reactivation of motor areas during self-triggered voluntary action might influence the perceptual signals from the arm and cause the SPL. Taken together, such views favor the hypothesis of an interaction between motor and somesthetic processes in SPLs.

If a polymodal perceptive interaction has to be retained, it is difficult to ignore a possible role of the visual system in the elicitation of this perceptive mismatch. In certain patients, vision can cancel phantom limbs or their associated features such as pain. ${ }^{27}$ Accordingly, one can reasonably assume that an aberrant visual coactivation may influence motor or polymodal areas, and thus play a role in the appearance of an SPL. Here, we report a clinical and fMRI investigation of a patient who suffered from a right subcortical stroke and experienced a SPL that she could feel, see, and intentionally move. Our aim was to assess the neural basis of this multimodal phenomenon using motor and sensorimotor tasks involving the SPL. According to the patient's reports, we asked her to scratch her right or left cheek with the SPL during functional imaging sessions. We hypothesized that scratching would require that she bring her SPL close to her eyes and, therefore, produce task-related activations in the visual and sensory cortices, reflecting the reportedly vivid phenomenal experience of her SPL.

\section{Subjects and Methods}

\section{Case Report}

The patient, a 64-year-old librarian with a medical history of treated hypertension and occasional depression, was admitted to the emergency department in March 2007 after a sudden onset of left-sided weakness and dysarthria. She had apparently driven a long distance in a hemiplegic state, and it was only on arrival at her destination, as she tried to walk, that her hemiplegia was noticed by a passerby, indicating the presence of anosognosia at least initially. At admission, her neurological evaluation showed an National Institutes of Health Stroke Scale score of 16. She was well oriented in time and place, cooperative, presented mild spatial neglect, initial left hemianopia, left facial and leg paresis, left arm plegia, hypoesthesia to pain, and severely decreased positional sense. The computed tomographic scan and MRI (see Results) confirmed a subcortical capsulolenticular hemorrhage. Behavioral changes included misoplegia, increased impulsivity, and emotional swing, as well as urinary incontinence during at least the first month of her hospitalization. Formal neuropsychological examination at 1 week (see Supplementary Table S1) excluded diffuse cognitive alteration and confirmed the presence of a left spatial neglect without signs of personal neglect. At this point, anosognosia had entirely resolved.

The intriguing symptom was the subjective appearance, from the 4th day on, of an SPL, starting from the elbow of the left plegic arm, which the patient claimed she could move, see, and even use to touch parts of her body (Fig 1; see supplementary materials for more details). Her SPL was not experienced permanently, but only when she decided to "trigger" it intentionally, thus meeting the rare defining feature of the "intentional motor phantom limb syndrome." 13 The SPL was visually perceived whenever it was "triggered." She described it as "pale," "milk-white," and "transparent." Tactile sensations in the SPL happened when she clenched her hand (she could then feel her phantom palm with her phantom fingers) and when she "touched" certain parts of her body (in which case, the sensation was felt both in the phantom and the touched body part). She could touch parts of her head, as well as her right shoulder. She claimed to be able to use the SPL to scratch an itch on her head (with an actual sense of relief). Moreover, she reported that the phantom could not penetrate solid obstacles (see supplementary materials for more details).

This phenomenon persisted during the following 4 months spent in the neurorehabilitation clinic and decreased progressively over the next few months, as she regained proprioceptive feelings and motor abilities in the plegic limb. At discharge, spatial neglect had completely receded, hemi- 


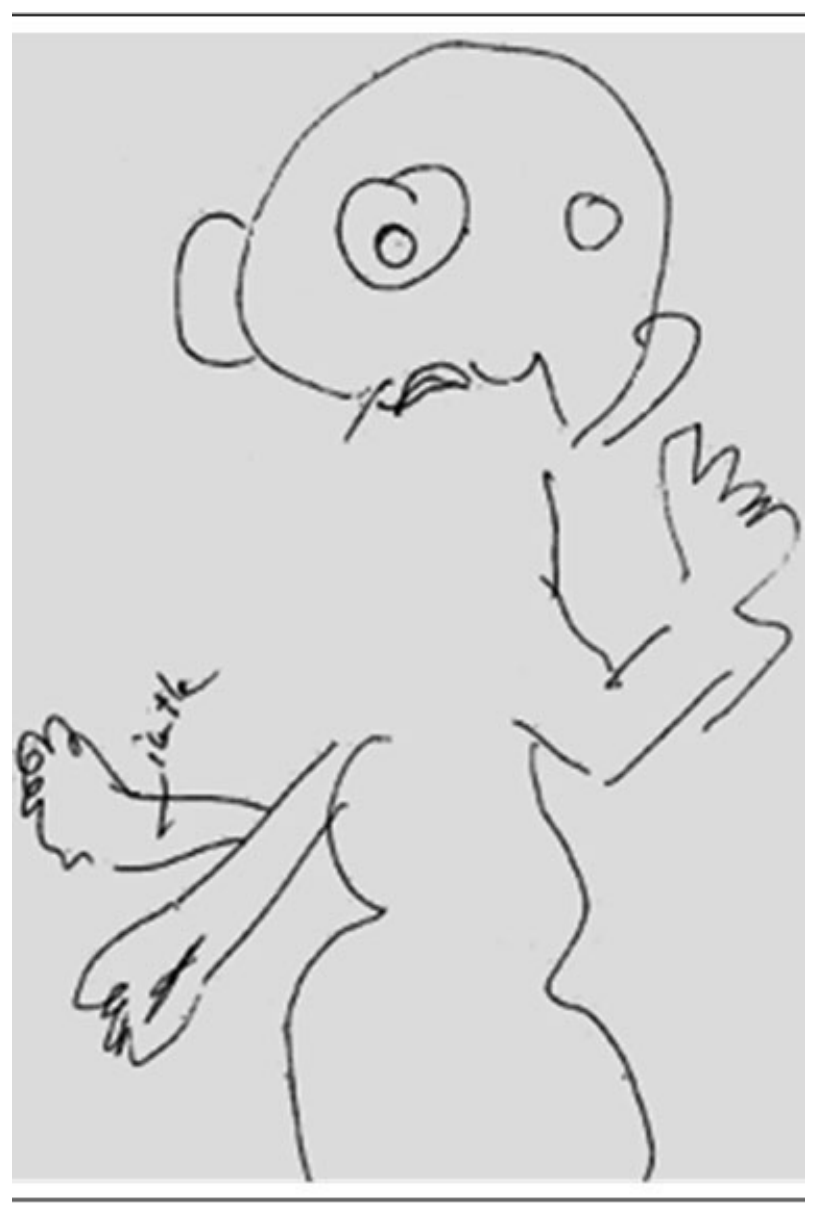

Fig 1. Schematic representation of the phantom limb as drawn by the patient. Note the left-right reversal of the body and the supernumerary phantom limb (SPL) emerging at the level of the elbow of the paralyzed arm, indicated here by the French word inutile (for "useless"; see additional notes in supplementary materials).

anopia turned into a quadrantopia, and hemiplegia partially recovered, particularly for the lower left limb.

\section{Functional Magnetic Resonance Imaging Investigation}

At 3 weeks after stroke, this study was designed to investigate the polymodal nature of the SPL. Our aim was to compare fMRI responses induced by real movements (RM) of the healthy right hand, imaginary movements (IM) of the right and left hands, and movements of the SPL, thus using the patient as her own control across conditions. Based on the patient's claim that she could see, move, and use her SPL, we predicted that SPL's movements might elicit activations in motor, somatosensory, and visual areas (see the paradigm detailed in the supplementary materials).

\section{Results}

\section{Behavioral Data}

The formal neuropsychological assessment conducted 1 week after stroke showed mainly cognitive difficulties related to spatial neglect (see Supplementary Table S1). With particular relevance to our study, when ques- tioned before the fMRI session, the patient still experienced her SPL, with no changes relative to previous clinical observations. After each MRI scan, the patient was systematically questioned about the feasibility of the task she had just performed (whether she saw and felt the SPL across conditions). Globally, she declared that she had performed the different tasks correctly. Thus, she could perform RM with the right hand and IM with both. When asked to perform movements with the SPL, she explained that, although she was able to scratch her left cheek correctly, she experienced some difficulties in scratching the right one because of the fact that the narrow space left by the scanner antenna "prevented" her SPL from reaching the right cheek, thus confirming her previous report that she was unable to move her phantom through obstacles.

\section{Functional Results}

SIMPLE MOTOR TASKS.

The fMRI results of simple motor tasks conducted with the healthy right and the paretic hand are summarized in Figures $2 \mathrm{~B}$ to D. The RMs of the right hand were, as expected, associated with dominant activation of the left sensorimotor (M1, S1) and premotor cortex (ventrolateral, dorsolateral, as well as medial premotor areas), left temporooccipital junction, left superior parietal lobule, and possibly a small contralateral hand representational cortex (see Fig 2B). Similar but less extensive activations were induced by the IMs of the same hand (see Fig 2C). More interestingly, the IMs of the paralyzed left hand activated the right motor and the bilateral dorsal premotor cortex, the supplementary motor area, and the left motor cortex (see Fig 2D and details in Supplementary Table S2).

\section{SENSORIMOTOR TASKS.}

Figure 2E illustrates the activation induced by the scratching of the right cheek by the SPL. Although the patient stated that she was unable to perform these movements correctly because of the scanner antenna, the activation involved the left and right hand/arm areas (M1), the premotor cortex, but also the left occipital cortex (BA 19/18, see details in Supplementary Table S2), compatible with the patient's experienced sensation of "moving and seeing" her SPL. Scratching the left cheek with the SPL (see Fig 2F), a task that she said was performed correctly, activated almost exactly the same premotor regions, the left occipital cortex, but here more extensively the right hand/arm M1 (as well as other areas detailed in the Table), again concordant with her report that she "moved and saw" her SPL. In contrast, scratching this same left cheek with the paretic left hand (see Fig 2G) activated the right motor and bilateral premotor areas (see the Table), but not the middle occipital areas. 


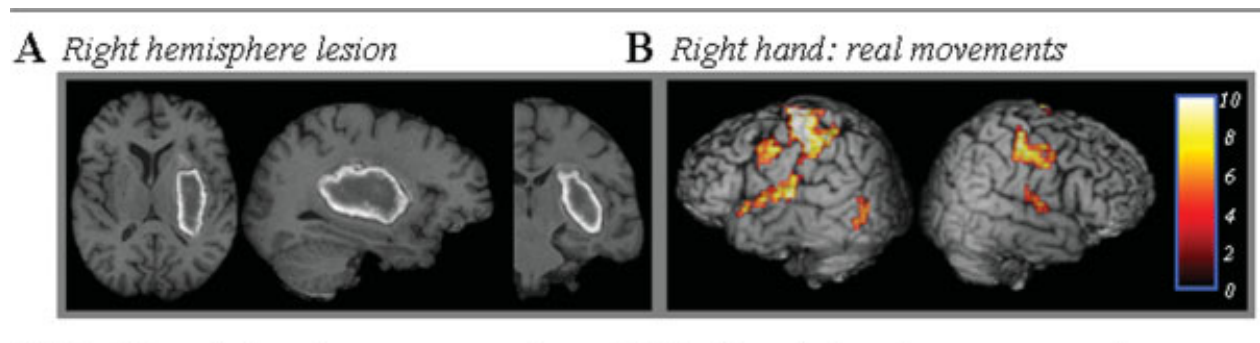

C Right hand: imaginary movements

D Left hand: imaginary movements
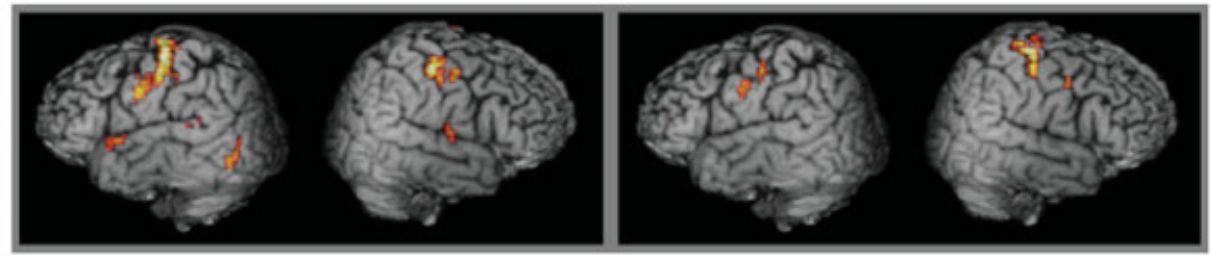

E SPL: right cheek

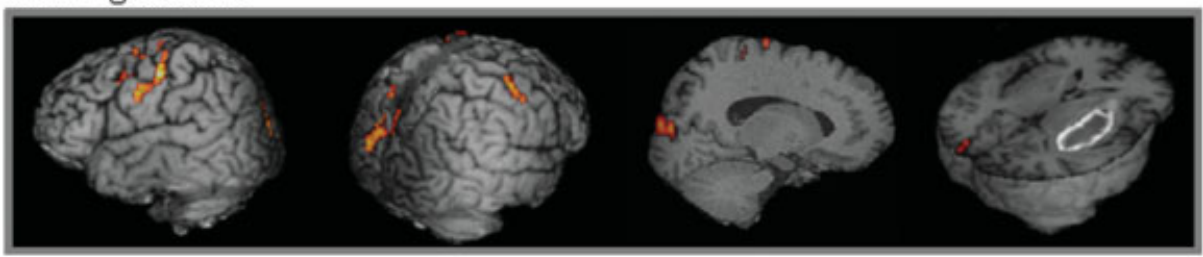

F SPL: left cheek

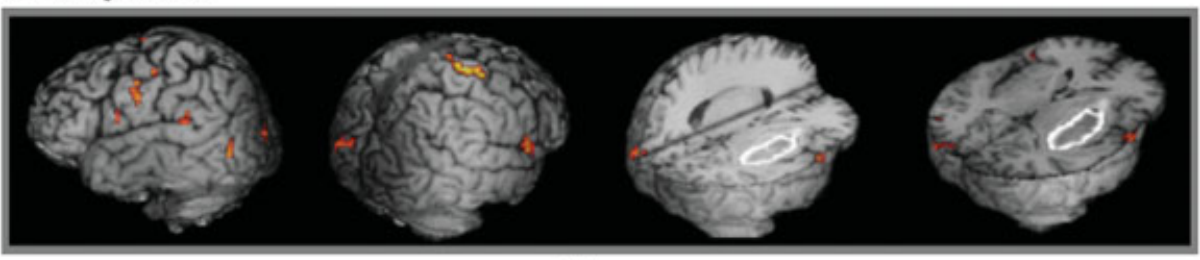

G Left hand: left cheek

H Left cheek: SPL vs left hand (r-G)

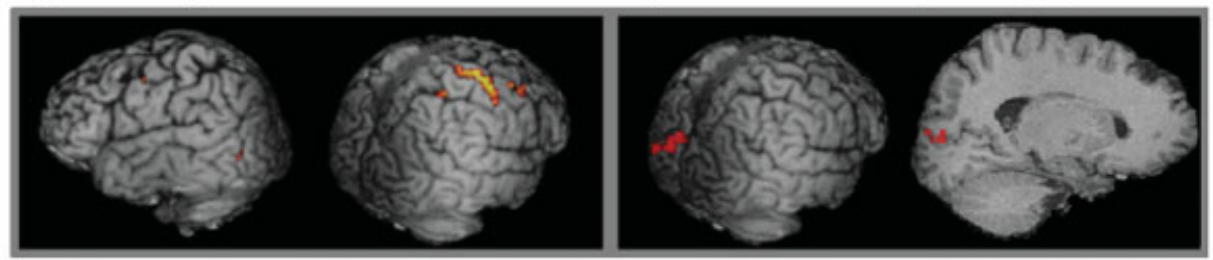

I SPL: left vs right cheek (F.E)

J Parameter estimates (contrasts B to G)

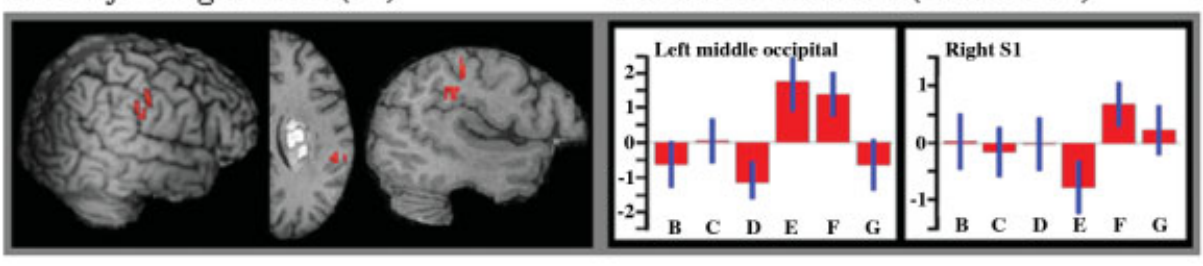

Fig 2. (A) Magnetic resonance imaging axial, sagittal, and coronal images showing the localization of the subcortical lesion. (B-D) Activation maps obtained respectively during real right hand, imaginary right, and left plegic hand movements. (E, $F)$ Activation maps for the supernumerary third hand movements scratching the right $(E)$ and the left cheek $(F)$. $(G)$ Imaginary left hand movements scratching the left cheek. (H) Comparison of left cheek scratching by the third phantom hand versus the left paralyzed hand (contrast illustrated in E minus G). (I) Comparison of the left cheek scratching versus the right cheek scratching with the third hand. (J) Parameter estimates of activation for two regions of interest over the different conditions illustrated in $(B-G)$. (J, left) Parameter estimates for the middle occipital gyrus found mainly in $(E)$ and $(F)$ in relation to the phantom movements. (J, right) Parameter estimates for the somesthetic S1 area demonstrated by the contrast in (I) (contrast F minus E). [Color figure can be viewed in the online issue, which is available at www.interscience.wiley.com.] 
Table. Activated Regions with Their Talairach $x, y, z$ Coordinates, Maximum Value of the t-Statistics of the Reported Cluster, the Cluster Sizes (K), and the Corresponding Brodmann Areas in the Sensorimotor Tasks

$\begin{array}{lllll}\text { Anatomic Location } & x, y, z & \mathrm{~T}_{\max } & \mathrm{K} & \text { BA }\end{array}$

SPL Scratching Left Cheek ${ }^{\mathrm{a}}$

Motor cortex

$\begin{array}{lcccc}\text { Right M1 (hand/arm areas) } & 31,-22,58 & 9.94 & 172 & 4 \\ - & 23,-35,62 & 8.48 & - & 4 \\ \text { Left M1 (hand area) } & -34,-29,49 & 6.51 & 11 & 4\end{array}$

Premotor cortex

\begin{tabular}{lcccc} 
Left SMA & $-11,-15,64$ & 8.25 & 103 & 6 \\
\hline Right SMA & $4,-14,61$ & 5.46 & - & 6 \\
\hline Left dorsolateral premotor cortex & $-45,-10,45$ & 7.07 & 61 & 6 \\
\hline- & $-47,1,26$ & 5.84 & 12 & 6 \\
Right ventrolateral premotor cortex & $55,0,12$ & 6.15 & 22 & 11 \\
Occipital cortex & & & 5.88 & 19 \\
\hline Left lingual gyrus & $-14,-57,-2$ & 5.26 & 27 & $19 / 18$ \\
\hline Left middle occipital gyrus & $-24,-96,13$ & 5
\end{tabular}

Other areas

Left superior temporal gyrus

$-50,-49,2$

7.39

23

$19 / 18$

Left temporoparietooccipital junction

$-41,-76,5$

7.05

Left inferior frontal gyrus

$-47,19,9$

5.91

$39 / 19$

Left Paretic Hand Scratching Left Cheek ${ }^{\mathrm{b}}$

Motor cortex

\begin{tabular}{|c|c|c|c|c|}
\hline Right M1 (hand/arm areas) & $31,-23,58$ & 10.21 & 162 & 4 \\
\hline Right M1 (arm area) & $18,-35,65$ & 5.65 & 11 & 4 \\
\hline \multicolumn{5}{|l|}{ remotor cortex } \\
\hline Right dorsolateral premotor cortex & $37,-1,48$ & 7.66 & 55 & 6 \\
\hline Left dorsolateral premotor cortex & $-35,-9,42$ & 5.33 & 10 & 6 \\
\hline \multicolumn{5}{|l|}{ ther areas } \\
\hline Left temporoparietooccipital junction & $-43,-70,8$ & 6.13 & 27 & $39 / 19$ \\
\hline Right superior parietal lobule & $31,-51,55$ & 5.76 & 17 & 7 \\
\hline
\end{tabular}

Left Cheek Scratching: SPL versus Paretic Left Hand ${ }^{\mathrm{c}}$

Occipital cortex

\begin{tabular}{|c|c|c|c|c|}
\hline Left lingual gyrus & $-13,-54,-1$ & 4.88 & 36 & 19 \\
\hline Left cuneus & $-5,-77,14$ & 4.37 & 161 & 18 \\
\hline Left middle occipital gyrus & $-14,-88,14$ & 4.31 & - & 19 \\
\hline \multicolumn{5}{|l|}{ ther areas } \\
\hline Left middle frontal gyrus & $-40,46,9$ & 5.75 & 30 & $46 / 10$ \\
\hline Left inferior frontal gyrus & $-47,23,9$ & 5.49 & 63 & 45 \\
\hline \multicolumn{5}{|l|}{ PL: Scratching Left versus Right Cheek $^{\mathrm{d}}$} \\
\hline ight somesthetic cortex (face area $\mathrm{S} 1$ ) & $47,-27,44$ & 4.50 & 23 & 1 \\
\hline- & $43,-34,28$ & 4.29 & 41 & $2 / 40$ \\
\hline
\end{tabular}

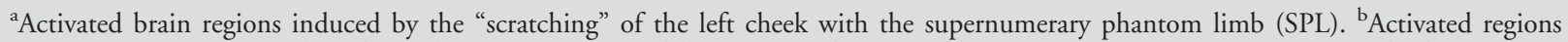
induced by "scratching" the left cheek with the plegic left hand. "Specific activation demonstrated by the comparison of left cheek scratching movements with the SPL versus the left plegic hand (ie, contrast the first section of the table minus the second section). ${ }^{\mathrm{d}}$ Activation of somesthetic areas demonstrated by the comparison of the left cheek scratching (said to be feasible by the patient) versus the right cheek (said to be unfeasible because of the magnetic resonance imaging scanner antenna preventing her to reach her cheek) with the SPL. Note that the first and second sections of the table refer to activation obtained from direct contrast of activation versus resting condition, whereas the third and fourth sections of the table refer to activation obtained from interaction analysis between two different contrasts (see details in supplementary materials). BA = Brodmann area; SMA = supplementary motor area. 
To further substantiate this later observation and assess the involvement of sensory cortex during scratching tasks, we conducted interaction analyses between the contrasts. The specificity of the occipital involvement during SPL movements was assessed by the comparison of activation induced by the scratching of the left cheek with the SPL versus the plegic hand (see Fig $2 \mathrm{H})$. This showed mainly significant voxels in the left middle occipital (BA 18/19; see the Table) and less extensively in prefrontal areas. Figure 2J (left), which illustrates the parameter estimates of activation for the middle occipital area, confirms that activity in this region was specifically related to SPL movements. Finally, the interaction between the scratching of the left cheek (declared as possible) versus the right cheek (impossible) by the SPL showed significant activation only in the right somesthetic S1 face area (see Fig 2I; see the Table). The parameter estimates displayed in Figure 2J (right) confirm that area S1 displayed the highest activation during left cheek scratching (see Fig 2J, lane F). This measurable sensory response suggests that the patient, indeed, experienced the sensation of left cheek scratching. However, although the "phantom touch" was reported as a "double touch" (ie, sensations both on SPL and the touched body part), the responses to such double-touch feelings are indistinguishable here, presumably because of the proximity of the hand and face receptive fields.

\section{Discussion}

This article describes the clinical features and neural correlates of a multimodal poststroke SPL characterized by the presence of motor, tactile, and visual components. The evidence presented here demonstrates a direct link between the sensorimotor characteristics of the illusion and modality-specific activations in motor, visual, and somesthetic areas. Although previous reports have described various clinical features of SPLs, few studies investigated the neural correlates of this phenomenon. In this respect, the case presented here is, to our knowledge, unique for its multimodal characteristics. Furthermore, this functional investigation appears to be the first to assess the neural basis of a multimodal stroke-induced SPL.

The visual characteristics of SPLs have not been discussed much in the stroke literature because the majority of such patients do not report seeing the phantom (nine visible SPL cases reported; see earlier). Even in the only other report of intentional motor SPL, the patient could move but not see the limb. ${ }^{13}$ Some SPLs are reported as not seen and not influenced, either by visual feedback of the actual limbs or direct touch of the actual (and reduplicated) limb. ${ }^{6,13}$ In the few cases of nonintentional SPLs, visibility was reported, although less precisely documented than here, and mostly in a delusional frame of mind. Inversely, in some instances, the SPL could even be cancelled by vision and tactile stimulation of the real limb. ${ }^{28}$ The presence of multimodal components in some cases suggests that these illusory phenomena might be better explained by a failure to correctly integrate multimodal and motor information to generate a normal experience of self-location ${ }^{29,30}$ than by sensorimotor reorganization as in amputees, ${ }^{31}$ although these two approaches are certainly not exclusive. The literature indeed suggests the existence of various types of SPLs differing according to the phenomenological richness (unimodal, bimodal, multimodal), degree of motor controllability (impossible, automatic, intentional movements, and so forth), sensitivity to sensory feedback (visual, tactile, proprioceptive), and patients' mental framework (confused, delusional, indifferent, lucid). In this respect, it appears unreasonable to expect a single explanation for such a clinically complex and diverse phenomenology.

Although rare, previous studies have assessed the neural correlates of the presence, or of the intentional movements, of stroke-induced SPL, ${ }^{13,26,28}$ but this investigation is the first to demonstrate the multimodal aspects of this phenomenon. Particularly, visual activation accompanying SPL movements has never been observed either in amputation ${ }^{32}$ or in poststroke phantoms. ${ }^{13,26}$ Also, the activation of somesthetic areas fits with the patient's reported conscious experience of feeling and seeing her SPL, thus reinforcing our opinion that she was not simply confabulating.

The activation of the left extrastriate areas involving the occipito-temporo-parietal junction appears explainable. This area has often been involved in the alteration of body schema perception. ${ }^{33-36}$ Its electrical stimulation was also found to induce illusory perception (proprioceptive and visual) of body part movements. ${ }^{34}$ Functional studies have also showed that the "extrastriate body area" responds to the perception of other people's body parts and to goal-directed movements (or imagined movements) of own body parts. ${ }^{37,38}$ Here, the activation coordinates correspond to those previously reported for the extrastriate body area $(x, y, z=$ $-45,-65,2$ in Peelen and Downing's article ${ }^{39} ; x, y$, $z=-48 ;-69 ; 6$ in Astafiev and colleagues' article ${ }^{37}$ ). In our study, although activation in this area was significant in some contrasts only (including RM and IM of the right hand; see Figs 2B, C), the analysis of the parameter estimates (not illustrated) showed its recruitment in all conditions and not only in relation to the SPL. Additional analysis in two control subjects showed the bilateral involvement of this area during $\mathrm{RM}$ of the right and left hand (unpublished data). Accordingly, we hypothesize that the perception of the flickering hand during the activation condition (vs the fixation during the resting condition) is the most plau- 
sible explanation for the involvement of this left temporoparietooccipital junction.

However, the activation found in the left occipital lobe was specifically related to the SPL movements, and involved several medial-posterior foci extending beyond the primary visual area V1, mostly area V2. ${ }^{40}$ Activity in such low-level visual areas (including the lingual gyrus and the cuneus) has been observed during visually guided eye and limb movements, ${ }^{37}$ but also in various other contexts. Thus, these areas were activated by motor imagery tasks, ${ }^{41}$ mental generation of words or objects images, ${ }^{42-44}$ and illusory contours. ${ }^{45}$ In light of these observations supporting the participation of low-level visual areas in contexts implying goaloriented movements, motor and mental imagery, the activation discussed here during "visualization" of the SPL's intentionally triggered and goal-directed movements appears to make sense. Finally, though the activation of these areas involved the bilateral visual cortex in control subjects, ${ }^{37,39}$ the lack of right activation in our patient might reasonably be explained by the initial hemianopia, possibly because of a hemorrhage-induced disconnection of these areas.

The anatomical disruption and the ensuing somesthetic deafferentation in our and other patients constitutes a necessary but not a sufficient condition for the appearance of an SPL. Existing evidence from strokeelicited SPLs convincingly implicates the mismatch between the subject's well-established sensorimotor representations and a suddenly aberrant pattern of communication between the brain and the paralyzed limb. In this respect, understanding of the phenomenological complexity of SPLs and the puzzles they pose could benefit from the examination of the general framework of the body schema alterations (eg, somatoparaphrenia, hemiasomatognosia, out-of-body experience, feeling of a presence, autoscopy, and heutoscopy; see Arzy and coauthors ${ }^{33}$ and Blanke and researchers' $\left.\operatorname{articles}^{34}\right)$. This view is concordant with Todd and Dewhurst's ${ }^{19}$ early statement that "the supernumerary phantom arm is in the nature of an autoscopic double of the real arm." However, given the unique characteristics of our patient's SPL, we are tempted to interpret it as a new neurological syndrome, filling the gap between unseen duplicated body parts in the peripersonal space and visible doubles of one's entire body in the extrapersonal space. In other words, and in line with other authors' views arguing for the continuity of disturbances from body parts to whole-body illusions, ${ }^{46,47}$ this case might constitute, at least conceptually and phenomenologically, a missing link between classic phantom limbs and autoscopic phenomena. Yet, the question remains why these two well-known phenomena are relatively frequent in nondelusional patients, whereas our patient's visible SPL is so unusual. Moreover, the questions of how disorders of the body schema, extending from isolated body parts to the entire body, are linked at the neurofunctional level and whether such disorders can ultimately fit a single explanatory model also remain moot points. Ultimately, however, these conditions might offer a unique way to understand how the brain constructs a normal experience of bodily awareness and the self.

This research was supported by the Swiss National Science Foundation (325100-118362) and the Center for Biomedical Imaging of Geneva and Lausanne (S.S.).

This article is dedicated to the memory of our friend and colleague neuropsychologist Eugène Mayer (James) who tragically passed away on December 14, 2008. We thank F. Henry for technical assistance.

\section{References}

1. Brugger P. From phantom limb to phantom body. In: Knoblich G, Thornton IM, Grosjean M, Shiffrar M, eds. Human body perception from the inside out. Oxford: Oxford University Press, 2006:171-209.

2. Halligan PW, Marshall JC, Wade DT. Three arms: a case study of supernumerary phantom limb after right hemisphere stroke. J Neurol Neurosurg Psychiatry 1993;56:159-166.

3. Weinstein EA, Kahn RL, Malitz S, Rozanski J. Delusional reduplication of parts of the body. Brain 1954;77:45-60.

4. Halligan PW, Marshall JC. Supernumerary phantom limb after right hemispheric stroke. J Neurol Neurosurg Psychiatry 1995; 59:341-342.

5. Brugger P. Supernumerary phantoms: a comment on Grossi, et al.'s (2002) spare thoughts on spare limbs. Percept Mot Skills 2003;97:3-10.

6. Vuilleumier P, Reverdin A, Landis T. Four legs. Illusory reduplication of the lower limbs after bilateral parietal lobe damage. Arch Neurol 1997;54:1543-1547.

7. Frederiks JA. Occurrence and nature of phantom limb phenomena following amputation of body parts and following lesions of the central and peripheral nervous system. Psychiatr Neurol Neurochir 1963;66:73-97.

8. Grossi D, Di Cesare G, Tamburro RP. On the syndrome of the "spare limb": one case. Percept Mot Skills 2002;94:476-478.

9. van Bogaert L. Sur la pathologie de l'image de soi. Ann Med Psychol 1934;92:519-555; 746-759.

10. Pinéas H. Ein Fall von phantomähnlichen Erscheinungen ("Phantomarm") bei hemiplegischer Lähmung. Nervenarzt 1932;5:233-236.

11. Bakheit AM. Phantom limb sensations after complete thoracic transverse myelitis. J Neurol Neurosurg Psychiatry 2000;69: 275-276.

12. Lhermitte F, Cambier J. Les perturbations somatognosiques en pathologie nerveuse. Paris: Masson, 1960:465-554.

13. Staub F, Bogousslavsky J, Maeder P, et al. Intentional motor phantom limb syndrome. Neurology 2006;67:2140-2146.

14. Bakheit AM, Roundhill S. Supernumerary phantom limb after stroke. Postgrad Med J 2005;81:e2.

15. Critchley M. The parietal lobes. London: Edward Arnold, 1953:255.

16. Hécaen H, de Ajuriaguerra J, Le Guillant L, Angelergues R. Délire centré sur un membre fantôme chez un hémiplégique gauche par lésion vasculaire avec anosognosie. Evol Psychiatr 1954;19:273-279. 
17. Lenz H. Anfälle von Verdopplung der rechten Körperhälfte sowie Verzerrung des Fühl- und Schaubildes rechts. Nervenarzt 1958;29:314-315.

18. Miyazawa N, Hayashi M, Komiya K, Akiyama I. Supernumerary phantom limbs associated with left hemispheric stroke: case report and review of the literature. Neurosurgery 2004;54: $228-231$.

19. Todd J, Dewhurst K. The double: its psychopathology and psychophysiology. J Nerv Ment Dis 1955;122:47-55.

20. Bakheit AM. Illusory movements of the paralysed upper limb in stroke. J Neurol Neurosurg Psychiatry 2000;69:837.

21. Hécaen $\mathrm{H}$, de Ajuriaguerra J. Méconnaissances et hallucinations corporelles. Paris: Masson, 1952.

22. Lohmann AM. Ein Fall von Phantomerscheinungen bei einer linksseitigen Hemiplegie. Dtsch. Z. Nervenheilk, 1935:136.

23. Mazzoni M, Lucchini C, Meucci G, et al. Supernumerary pianto limb after ischaemic stroke. Neurocase 1997;3:223-230.

24. Sellal F, Renaseau-leclerc C, Labrecque R. [The man with 6 arms. An analysis of supernumerary phantom limbs after right hemisphere stroke]. Rev Neurol (Paris) 1996;152:190-195.

25. Frith CD, Blakemore SJ, Wolpert DM. Abnormalities in the awareness and control of action. Philos Trans R Soc Lond B Biol Sci 2000;355:1771-1788.

26. McGonigle DJ, Hanninen R, Salenius $S$, et al. Whose arm is it anyway? An fMRI case study of supernumerary phantom limb. Brain 2002;125:1265-1274.

27. Giraux P, Sirigu A. Illusory movements of the paralyzed limb restore motor cortex activity. Neuroimage 2003;20(suppl 1): S107-S111.

28. Hari R, Hanninen R, Makinen T, et al. Three hands: fragmentation of human bodily awareness. Neurosci Lett 1998;240: 131-134.

29. Blanke O, Landis T, Spinelli L, Seeck M. Out-of-body experience and autoscopy of neurological origin. Brain 2004;127: 243-258.

30. Brugger P. Reflective mirrors: perspective-taking in autoscopic phenomena. Cogn Neuropsychiatry 2002;7:179-194.

31. Ramachandran VS, Rogers-Ramachandran D. Phantom limbs and neural plasticity. Arch Neurol 2000;57:317-320.

32. Roux FE, Lotterie JA, Cassol E, et al. Cortical areas involved in virtual movement of phantom limbs: comparison with normal subjects. Neurosurgery 2003;53:1342-1353.
33. Arzy $S$, Seeck $M$, Ortigue $S$, et al. Induction of an illusory shadow person. Nature 2006;443:287.

34. Blanke O, Ortigue S, Landis T, Seeck M. Stimulating illusory own-body perceptions. Nature 2002;419:269-270.

35. Coslett HB. Evidence for a disturbance of the body schema in neglect. Brain Cogn 1998;37:527-544.

36. Halligan PW, Marshall JC, Wade DT. Unilateral somatoparaphrenia after right hemisphere stroke: a case description. Cortex 1995;31:173-182.

37. Astafiev SV, Stanley CM, Shulman GL, Corbetta M. Extrastriate body area in human occipital cortex responds to the performance of motor actions. Nat Neurosci 2004;7:542-548.

38. Downing PE, Jiang Y, Shuman M, Kanwisher N. A cortical area selective for visual processing of the human body. Science 2001;293:2470-2473.

39. Peelen MV, Downing PE. Selectivity for the human body in the fusiform gyrus. J Neurophysiol 2005;93:603-608.

40. Tootell RB, Hadjikhani NK, Vanduffel W, et al. Functional analysis of primary visual cortex (V1) in humans. Proc Natl Acad Sci U S A 1998;95:811-817.

41. Bakker M, De Lange FP, Helmich RC, et al. Cerebral correlates of motor imagery of normal and precision gait. Neuroimage 2008;41:998-1010.

42. Kosslyn SM, Thompson WL, Kim IJ, Alpert NM. Topographical representations of mental images in primary visual cortex. Nature 1995;378:496-498.

43. D'Esposito M, Detre JA, Aguirre GK, et al. A functional MRI study of mental image generation. Neuropsychologia 1997;35: $725-730$.

44. Slotnick SD, Thompson WL, Kosslyn SM. Visual mental imagery induces retinotopically organized activation of early visual areas. Cereb Cortex 2005;15:1570-1583.

45. Seghier ML, Vuilleumier P. Functional neuroimaging findings on the human perception of illusory contours. Neurosci Biobehav Rev 2006;30:595-612.

46. Wade NJ. Beyond body experiences: phantom limbs, pain and the locus of sensation. Cortex 2008;5:5.

47. Brugger P, Regard M, Landis T. Illusory reduplication of one's own body: phenomenology and classification of autoscopic phenomena. Cogn Neuropsychiatry 1997;1:19-38. 